\section{PERSPECTIVAS ONTOSEMÁNTICAS EN LA CONCEPCIÓN ESTRUCTURALISTA DE LA CIENCIA}

\section{ONTOSEMANTICAL PERSPECTIVES IN THE STRUCTURALIST VIEW OF SCIENCE}

Adriana Gonzalo

Universidad Nacional del Litoral-CONICET Argentina

\begin{abstract}
Some reflexions about the ontosemantic problems in the Structuralist View of Science (SV) are introduced. They are presented from a historical perspective, beginning with Sneed (1971) and Stegmüller (1973, 1976), up to more recent works of Moulines (1982, 1984, 1989, 1994, 1998, 2002).

The particular ontosemantic problems here considered are: the distinction between the $T$-theoretical terms (relative to a theory $T$ ) and the $T$-non-theoretical terms; and the criteria of meaning for scientific terms. The solutions given in the frame of the SV are analyzed and discussed form a critical perspective in order to evaluate its successes and limitations.
\end{abstract}

KEY WORDS: Ontosemantics; scientific theories; structuralist view.

1. Introducción. Respecto de los problemas ontosemánticos en la ciencia, muchos autores han hecho hincapié en los aspectos gnoseológicos implicados en éstos: la actividad sensorial, los procesos perceptivos, la elaboración perceptual-conceptual. Mientras que otros autores intentaron penetrar particularmente los aspectos lógicos y semánticos, postulando que sin el conocimiento de éstos, no sería posible el esclarecimiento de aquellos problemas. Frege aparece aquí a la cabeza de una tradición que priorizó el esclarecimiento del problema de la significación de determinadas expresiones al de cómo es posible el conocimiento de éstas'.

Coffa, quien ha caracterizado la perspectiva anterior como "tradición semántica", afirma respecto del viraje fregeano:
RESUMEN: El presente trabajo analiza las reflexiones llevadas a cabo en el seno de la Concepción Estructuralista (CE) en relación a una agenda de los principales problemas ontosemánticos de las teorías científicas planteados en ese marco metateórico. Se presenta un raconto histórico del desarrollo de las problemáticas y de las soluciones teóricas brindadas en el devenir de la CE, partiendo de Sneed (1971) y Stegmüller $(1973,1976)$, hasta los trabajos de Moulines (1982, 1984, 1989, 1994, 1998, 2002).

El trabajo se centra en el problema de distinción entre términos T-teóricos y T-no-teóricos, y en los criterios de significación involucrados. Asimismo, se analiza la solución pragmática de la CE en la determinación del significado de los términos científicos, con el objetivo de evaluar aciertos y límites de la propuesta programática.

PALABRAS CLAVE: Ontosemántica; teorias científicas; concepción estructuralista.

\begin{abstract}
A lo largo del siglo XIX, observamos un desarrollo muy complejo de la semántica, al cabo del cual se hace posible reformular la doctrina kantiana de la constitución de una manera defendible: Kant está en lo cierto al señalar que la mente humana desempeña un papel decisivo en el conocimiento al constituir algo, pero se equivoca al pensar que lo constituido son los objetos de los que tenemos conocimiento y experiencia. Lo construido en cambio son los conceptos en términos de los cuáles pensamos el mundo o, en otras palabras nuestros significados, nuestro lenguaje, nuestro marco conceptual: no aquello de que hablamos sino lo que podemos decir acerca de ello (Coffa, 1991, 19)
\end{abstract}

Gran parte de los trabajos sobre la ontosemántica de la ciencia presentados en el marco de la Concepción Estructuralista (CE) se inscriben en esa perspectiva, particular- 
mente los trabajos de Moulines (1984, 1994, 1998, 2002). Leemos, por ejemplo, en Moulines (2002):

La pregunta esquemática fundamental: (1) ¿Hay esto? Debe ser complementada con otras dos preguntas esquemáticas igualmente fundamentales: (2) ¿Cómo puedo saber que hay esto? Así como: (3) ¿Qué significa "esto"? De hecho la pregunta (1) depende metodológicamente de la pregunta (2) y ambas, a su vez, de (3). [...] Creo que el primer filósofo en haberse percatado plenamente de la mutua imbricación de las preguntas (1), (2) y (3), asi como de la preeminencia metodológica de (3), fue Gottlob Frege. Por ello podemos considerarlo como el pionero avant la lettre de la disciplina que llamo "ontoepistemosemántica" (Moulines, 2002, 1).

Pero, además, a lo largo del desarrollo de la CE se ha sostenido que debe admitirse la presencia de componentes pragmáticos en la determinación del significado, posición que ha sido defendida desde los trabajos iniciales de Sneed (1971) y Stegmüller (1973).

En especial, en los trabajos señalados de Moulines, como también los propios de Balzer, se han presentado tratamientos bastante pormenorizados de lo que podríamos denominar una "estrategia semántica". Sin embargo, contrariamente a la elucidación lógico-semántica de los problemas de la significación de los términos y expresiones en las teorias científicas; la profundización sobre los aspectos pragmáticos del significado es una tarea poco desarrollada, y tal vez, podríamos decir: una tarea a realizar en el seno de la CE.

En el presente trabajo me propongo presentar y comentar la propuesta ontosemántica de la $C E$, y evaluar los aciertos y límites de la misma, en particular, como señalé antes, en lo relativo a la solución pragmática relativa a la significación de los términos científicos.

2. La CE, como parte de las denominadas "concepciones semánticas" de la ciencia, comparte con éstas la visión general de que las teorías deben ser concebidas como conjunto de modelos, que se aplican a ciertos dominios físicos o empíricos; en lugar de pensar en las teorías como conjunto de enunciados, o tipos de entidades lingüísticas. También es común a las concepciones semánticas pensar la noción de "modelo", ligada a la definición tarskiana de modelo, y sostener la idea de que una cierta estructura dada en lenguaje formal básico (generalmente del orden de la matemática, más que el de algún lenguaje formal lógico) expresa o representa componentes de una teoría; que puede aplicarse a un cierto dominio empírico.

Dentro de este marco genérico, desde la CE se concibe que la ciencia empírica en un tiempo dado consiste en un número grande y finito de unidades elementales que se denominan "elementos-teóricos". Éstos son caracterizados como entidades modelo-teóricas, clases de estructuras teoréticas de conjunto. Para describir estas entidades se introduce un aparato técnico específico, haciendo uso de una axiomatización basada en la teoria intuitiva de conjuntos. Un elemento-teórico, se representa como $\mathrm{T}=\langle\mathrm{K}, \mathrm{I}\rangle$, donde $\mathbf{K}$ es el núcleo teórico de $\mathrm{T}$, e I representa el conjunto de aplicaciones propuestas o intencionales (intended applications) del elemento-teórico.

Entre los elementos de $\mathbf{K}$, encontramos un conjunto de modelos. El papel de las entidades modélicas es clave y central en $C E$, esto se ve claramente en la necesidad de postular los diferentes tipos de modelos: (i) actuales; (ii) posibles o potenciales; (iii) potenciales parciales. Los tipos de modelos comparten la caracteristica de ser entidades de tipo lógico-representacional, que no forman parte de las teorias (objeto de las reconstrucciones), ni pertenecen al mundo fáctico. Estas entidades resultan instrumentalmente fructiferas en las reconstrucciones teóricas, y logran ser adecuadas para señalar el vínculo entre teorías y realidad extra-teórica o realidad empírica.

Precisando mejor los componentes de $\mathbf{K}$, diremos que un modelo potencial $\left(M_{p}\right)$ se caracteriza como una entidad determinada por las condiciones estructurales que intervienen en la caracterización del predicado conjuntista correspondiente a la reconstrucción formal de la teoria en cuestión. Intuitivamente, $M_{p}$ de una teoría dada $T\left(M_{p}(T)\right.$ ) es cualquier sistema del que sabemos que tiene la estructura conceptual requerida para ser un modelo de $\mathrm{T}$. Seguidamente, si a las condiciones que determinan los $M_{p}$ de $T$ añadimos un conjunto de axiomas o leyes generales de $T$, entonces las entidades que satisfacen éstos son denominadas los modelos actuales de $\mathrm{T}(\mathrm{M}(\mathrm{T}))$. Como dijimos, $\mathrm{K}$ contiene también el conjunto de los modelos potenciales parciales de una teoría $T\left(M_{p p}(T)\right)$. Estas estructuras describen mediante términos (llamados técnicamente "términos no-teóricos" -en el sentido en que se precisará luego-) los sistemas posibles a los que es concebible aplicar K. Así, 
podemos afirmar que los $\mathrm{M}_{\mathrm{pp}}(\mathrm{T})$ constituyen lo que podría llamarse la "base empírica" de T.

De los tipos de modelos comentados, sólo los $\mathrm{M}(\mathrm{T})$ serian compartidos con las otras metateorias de la tradición semántica. Los $\mathrm{M}_{\mathrm{pp}}(\mathrm{T})$ podrían de algún modo acercarse a lo que en Dalla Chiara y Toraldo di Francia (1999) se Ilama "modelos de datos" (aunque la asimilación no es sino aproximada). Por su parte, la idea de $\mathrm{M}_{\mathrm{p}}(\mathrm{T})$ es ciertamente un aporte original de la $C E$, y constituye un elemento básicamente formal, ya que en éstos se hace constar todos los elementos que componen el aparato conceptual necesario para establecer los modelos de la teoría en cuestión, a aplicarse a algún ámbito de una realidad empírica.

Avancemos ahora sobre el modo en que se conforma el aparato conceptual expresado en $\mathrm{M}_{\mathrm{p}}(\mathrm{T})$. En este sentido, se pensó tradicionalmente que la teoría intuitiva de conjuntos ofrecía un lenguaje potencialmente apropiado, y se concibió que los elementos de $\mathrm{M}_{\mathrm{p}}(\mathrm{T})$ se expresarian en términos de conjuntos, relaciones y funciones. A su vez, éstos recibirian ciertas caracterizaciones y tipificaciones, tales comos propiedades de los conjuntos, de las relaciones y de las funciones. Nótese entonces que la capacidad expresiva de los $M_{p}$ debe quedar circunscripta a los tipos de entidades conjuntistas señaladas. Consecuentemente los $\mathrm{M}(\mathrm{T})$ (las leyes de la teoría) serán expresados mediante un conjunto de conceptos (un subconjunto de los conceptos de $M_{p}(T)$ del tipo descrito.

Por su parte, los $M_{p p}(T)$ son entidades cuya capacidad expresiva también viene determinada en los $\mathrm{M}_{\mathrm{p}}(\mathrm{T})$. Es necesario, entonces, fijar la diferencia entre los conceptos característicos de los $M(T)$ y los de los $M_{p p}(T)$; porque, si bien los conceptos de ambos tipos de entidades están limitados o circunscritos al aparato conceptual definido en $M_{p}(T)$, es claro que se quiere metateóricamente diferenciar entre modelos reales y modelos potenciales parciales de un elemento teórico T en cuestión.

Cabe aclarar que, sumado a los componentes mencionados, en la CE se sostiene que una teoría tiene muchos modelos, y que los mismos deben estar interconectados formalmente, conformando una estructura global, por lo que se propuso la idea de constraints (C), como condiciones de ligadura entre las funciones correspondientes a los diversos modelos de una teoría. Además, dado que las teorías no son entidades aisladas, sino que, por el contrario, se pueden identificar diversas "familias"de teorías, que pueden reconstruirse sobre la base de relaciones interteóricas (reducción, especialización, equivalencia, etc.), resultó necesario pensar en links interteóricos (L), que conectaran estas teorias emparentadas. De modo que $\mathbf{K}$ quedaría así conformado: $K=\left\langle M_{p}, M, M_{p p}, C, L\right\rangle$.

El núcleo matemático concebido de este modo, se utiliza para realizar aserciones empíricas de la teoría. La expresión "aserción empírica", usada originalmente por Sneed (1971), se derivó de una idea característica de la física matemática: "applications". Las aserciones empíricas afirman que el predicado conjuntista se aplica sobre sistemas físicos, conformando de este modo el conjunto de las aplicaciones propuestas (o intencionales), características de la teoría. Según la $C E$, podemos reconocer diferentes aplicaciones de la misma teoría identificando el mismo formalismo matemático que es usado para realizar aserciones sobre diferentes sistemas físicos en el conjunto de las aplicaciones propuestas. Para que esto sea posible, es necesario que se hayan conceptualizado estos sistemas a los que se desea aplicar el formalismo del núcleo en términos compatibles con la conceptualización presentada en éste. De este modo, se concibió que estos sistemas debieran considerarse como estructuras del tipo de los modelos parciales de la teoría, estructuras que son compatibles con la teoría, pero que no la presuponen. Por ende, se definió el conjunto de las aplicaciones propuestas de una teoría, que designamos con $\mathbf{I}$, como incluido en el conjunto de los $\mathbf{M}_{\mathrm{pp}}$ de T. [Posteriormente, en Balzer, Moulines \& Sneed (1987) se precisará que $\left.\mathbf{I} \subseteq \mathrm{Po}\left(\mathrm{M}_{\mathrm{pp}}\right)\right]$.

3. Como hemos señalado rápidamente antes $-y$ ahora pasamos a precisar- los conceptos propios de los $\mathrm{M}_{\mathrm{pp}}(\mathrm{T})$ son denominados "términos no teóricos relativos a la teoría T en cuestión", o más sintéticamente: "términos T-no-teóricos". Notemos que estamos aquí frente a un corte que caracteriza qué es aquello propio de una teoría T, y qué es aquello que una teoría menta, pero no es propio (no constituye la identidad de la misma). Y esta línea demarcatoria se trazará sobre la distinción semántica: "término T-teórico"/"término no-T-teórico". Vamos pues a revisar como se presenta esta distinción.

Las distinción central en la CE: término T-teórico y término T-no-teórico fue presentada originalmente en Sneed 
(1971), donde se proponía el denominado "criterio de teoricidad" (CT). La idea que estaba en la base del criterio es una intuición sobre la práctica científica. Informalmente, podriamos explicitarlo así: existe una teoría dada $\mathbf{T}$ y un grupo de científicos que operan con T. En el caso de conceptos que son funciones o relaciones, se requiere conocer algunos valores de éstas relativos a $\mathrm{T}$ para determinados argumentos dados. Este conocimiento no puede adquirirse por "observación directa", sino que se requieren experimentos y mediciones que se llevan a cabo en orden a determinar los valores deseados. Todas estas actividades, que dan como resultado uno o más valores de las funciones identificables, representan las determinaciones para algún término.

Teniendo en cuenta ejemplos concretos de tales determinaciones reconocemos que, en el curso de una determinación, los científicos en general usan conocimiento teórico, fórmulas, ecuaciones. Éstos realizan ciertos cálculos e inferencias sobre la base de fórmulas dadas de modo de obtener valores de la función que se desea conocer. Usualmente, este conocimiento teórico se da en un contexto concreto sin mayor justificación: se lo asume o presupone. Todos los tipos de afirmaciones teóricas usadas en el curso de una determinación se consideran "presuposiciones". Para cualquier tipo de presuposición (una ecuación, una fórmula, etc.) se dan dos casos posibles: 0 se da que proviene (es parte) de alguna teoría T' diferente de $T$, o es parte de, o idéntica a $\mathbf{T}$ (vale decir, coincide con los axiomas de T). Para determinar o calcular por ejemplo las masas de las partículas elementales, los físicos usan la versión relativista de la ley de conservación del momento, que es la parte central de la mecánica relativista de colisión; para determinar los ángulos de las trayectorias de las partículas luego de una colisión usan las fórmulas de la geometría física, que es una teoría diferente de la mecánica de colisión. Sneed sostiene que esta distinción de si las teorías presupuestas en una determinación de algún término son todas idénticas a T o no; es equivalente a la distinción entre términos T-teóricos y T-no-teóricos.

Así resulta el CT: que un término $t_{i}$ de $\mathrm{T}$ es T-teórico si en todas las determinaciones de $t_{i}$ las partes de la teoría usada (presupuesta) por los científicos con el propósito de determinación son parte o coinciden con T. En otras palabras, los términos T-teóricos son términos que pueden ser determinados únicamente por medio de $T$.
4. Si bien el criterio sneediano representa una concepción pragmática de teoricidad, Sneed sólo esbozó la misma; y a pesar de la riqueza potencial de su planteo, cierto es que el escaso desarrollo y precisión que las ideas de "disponer de una teoria", "determinaciones" (de los términos de una teoría) y "presuposiciones" (en los procesos o procedimientos de determinación de un término relativo a una teoría) dejaron abiertas muchas criticas e interrogantes.

El trabajo de Sneed (1971) tuvo recepción y contribuyó centralmente a su difusión a través del impacto de la obra de Stegmüller (1973). Éste presentaría una versión menos técnica y con mayor desarrollo filosófico de lo que el autor presentara como una concepción no-enunciativa de la ciencia (en oposición a la concepción enunciativa propia de la Concepción Heredada de las teorias científicas), y que posteriormente llamaría "concepción estructuralista" de la ciencia. Stegmüller profundizaba alli algunas nociones semánticas bosquejadas por Sneed.

La noción de "disponer de una teoría" fue desarrollada en Stegmüller (1973, 1976 entre otros). En Stegmüller (1976, 222-223) el autor afirmaba que para la caracterización de la expresión se requería de conceptos extralógicos, como los de "persona", "creer que", "tener evidencia de apoyo para", así como una variable temporal $t$. Estos conceptos se enmarcaban en el terreno de las actitudes proposicionales de sujetos pertenecientes a una comunidad científica en un tiempo $t$ determinado, de modo que "la persona $p$ cree que $Y$ " y "p tiene evidencia empírica a favor de $Y$ " en un tiempo $t^{2}$. Según el autor, estas nociones escapaban a una precisión formal, y requerian de un tratamiento pragmático.

En Balzer \& Moulines (1980) y en Moulines (1985) ${ }^{3}$ se propusieron algunas modificaciones y precisiones al criterio de teoricidad sneediano modificándolo del siguiente modo:

Sea $f$ un término fundamental en $\mathrm{T}$ (esto es, $f$ no puede definirse por medio de otros términos de T). Si se supone que T es una teoría científica, entonces la determinación de la extensión de $f$ en $\mathrm{T}$ no será arbitraria (...), sino que se seguirán algunos patrones de determinación especificables, intersubjetivos y repetibles. Cuando fes un concepto métrico esos patrones son lo que normalmente se llama "métodos de medición" (Moulines, 1985, 473). 
Sin embargo, se percibió que si este criterio se circunscribía a las teorias que contuviesen términos métricos, resultaria insuficiente para caracterizar a otras teorias, que poseyesen sólo términos cualitativos. Así, se introdujo la expresión "métodos de determinación", y se afirmaba que: "Si se denomina $\mathbf{M} f(\mathbf{T})$ al conjunto de todos los modelos potenciales de $\mathrm{T}$ que se usan como métodos para determinar $f$, entonces la idea original de Sneed puede hacerse más precisa por medio del siguiente "criterio de teoricidad" (CT'): $f$ es T-teórico si $\mathbf{M} f(\mathbf{T}) \subseteq \mathbf{M}(\mathrm{T}) "$ (Moulines, 1985, 473). "Ahora bien, admitiendo que en una teoria científica T siempre encontraremos algún término para el cual existe algún método de determinación dentro de $\mathrm{T}$ que no es un modelo de T, se llama T-no-teóricos a tales términos" (Moulines, 1985, 474).

Un problema que Sneed había dejado abierto es el de si todas las teorías científicas constan de términos T-noteóricos. La propuesta de Moulines establece una "condición de vinculación de los términos T-no-teóricos" (LNT), mediante la cual relaciones o vínculos interteóricos (links) garanticen la existencia de métodos de determinación externos a una teoría $T$, vale decir, que posibiliten determinar los términos T-no-teóricos relativos a $\mathrm{T}$. Uno de los ejemplos más citados es la no-teoricidad de la función posición en la mecánica newtoniana de partículas, que se asocia al hecho de que la posición se vincula con las funciones de longitud y ángulo de la geometría física, y existen métodos geométricos de determinación de la longitud y del ángulo que no presuponen a las leyes de la mecánica newtoniana.

"El objetivo de la condición de LNT es inducir la formulación de la hipótesis metaempírica de que todos los términos no-teóricos de cualquier teoría científica están esencialmente vinculados con algunos términos de otras teorías" (Moulines, 1985, 477). De cumplirse esta condición, más el criterio $\mathrm{CT}^{14}$, tendriamos lo que el autor llama "términos no-teóricos normales". Moulines se pregunta si podría haber términos no-teóricos "anormales" en la ciencia. Éste es un problema significativo, y como hace notar el autor, si todos los términos-no-teóricos en T satisfacen para alguna teoría diferente $\mathbf{T}_{\mathrm{i}}$ la $L \mathrm{LNT}$, entonces el sentido de "teórico" podría extenderse y decirse que son teóricos en un sentido derivado. Esto conduciría a que no nos podríamos salir de una especie de macro red con vínculos interteóricos, y perderíamos la posibilidad de ha- blar de datos empíricos extralingüísticos o extramodélicos. Seguidamente, perderíamos la posibilidad de pensar una justificación teórica sobre "bases externas" a la teoría en cuestión, y deberíamos inclinarnos a favor de un criterio coherentista de justificación en la ciencia.

Sin embargo, pareceria que ni en los planteos tradicionales de Sneed y Stegmüller, ni tampoco en las elaboraciones posteriores de Moulines, se esté dispuesto a pensar que la salida al problema sea de tipo coherentista. Al admitir LNT, se está exigiendo que existe una teoría y un modo de determinación del significado de un T-teórico distinto de $T$, de este modo, se puede contar con la posibilidad de determinar los $\mathbf{M}_{\mathrm{pp}}$ de una teoría $\mathrm{T}$ en cuestión, como constituyendo la base empírica de T. Así, seguiriamos sosteniendo que para que una teoría científica pueda diferenciarse de una teoria ficcional debe existir algún tipo de entidades extralingüisticas de las que podamos dar cuenta sin presuponer en todo momento T. Esta manera de hablar de "entidades extralingüisticas" puede ampliarse cuando extendemos LNT como condición a un conjunto de teorías relacionadas entre sí por un conjunto de links, y/o relaciones interteoréticas, situación que resulta particularmente clara cuando hablamos de red teórica.

5. Pasemos ahora a señalar algunos límites en la concepción ontosemántica de la CE. En primer lugar, la propuesta se restringe al significado de los términos en la ciencia. No se presenta una teoría general del significado; ni se pretende -como sucedía en la Concepción Estándar- brindar una teoría que abarque a todo el conocimiento en general.

En segundo lugar, la propuesta se circunscribe a los significados de los términos o expresiones en la ciencia sobre la base de presentar la distinción T-teórico/T-no-teórico como esencial al aparato metateórico de la CE.

Seguidamente, dado que -al menos en la propuesta tradicional- los términos de una teoría se corresponden con entidades conjuntistas, propias de la axiomatización, la teoría semántica se restringe a términos asociables a nociones conjuntistas: conjuntos, relaciones, funciones.

La distinción comentada se estima sobre un criterio "pragmático" de teoricidad en la mayor parte de las conceptualizaciones en el seno de la $C E$, criterio que intenta constituirse como una teoria pragmática del significado 
de los términos científicos. Sin embargo, como haremos notar, esta teoría pragmática del significado de los términos científicos se esboza en la $C E$, pero no se desarrolla, y constituye -creo- una asignatura pendiente.

Hemos comentado antes la aproximación a la pragmática del significado llevada a cabo en Sneed (1971), Stegmüller (1973, 1979), Balzer \& Moulines (1980), Moulines (1985). Voy a detenerme en lo que sigue a comentar algunos aspectos de la propuesta posterior de Moulines.

En Moulines (2002) se afirma:

La semántica que tengo en mente, y que es componente fundamental de la ontoepistemosemántica, es una semántica que engloba la pragmática, al menos en la medida en que esta última sea relevante para las preguntas (1) (2) y (3) [anteriormente citadas]. Precisamente concibo lo que se puede llamar el aspecto no-referencial del significado de las expresiones como esencialmente pragmático (Moulines, 2002, 1-2).

Más adelante el autor nos dice: "[...] y esta última [(3)] depende en parte, Wittgenstein dixit, de los usos, o sea, de la pragmática de los términos de las teorías" (Moulines, 2002, 4).

En efecto, Moulines planteaba al comienzo del texto referido: "Podemos (y debemos, según creo) colorear la semántica básicamente fregeana con un matiz wittgensteineano. Wittgenstein nos enseñó que (en una gran clase de casos) 'el significado de una palabra es su uso en el lenguaje'5" (Moulines, 2002, 2).

Explicitando su posición, Moulines sostiene: "Mi punto de partida es fregeano -modificado por Tarski (para el aspecto referencial) y por Wittgenstein (para el aspecto no-referencial)" 6 .

Los que estamos familiarizados con la $\mathrm{CE}$, recibimos como claramente accesible el acercamiento entre la CE y la noción fregeana de referencia. Pero, a algunos al menos, nos cuesta comprender la idea de "aspectos no-referenciales del significado" asociada a la idea wittgensteiniana de "uso"; aún más cuando se afirma: "Estos usos son (parte de) los 'sentidos' de los términos según el significado originario que Frege dio a 'sentido'" (Moulines, 2002, 3).
En Moulines (1991) se habia ya indagado sistemáticamente la distinción global entre sentido y referencia. La lectura del texto me sugiere la idea de que hay una identificación entre sentido-referencia y aspectos no-referenciales del significado-referencia; ya que "sentido" de Frege se interpreta en parte pragmáticamente (a lo Wittgenstein) y en parte semánticamente (incluyendo los aspectos modales y otros) $y$, por ende, es una expresión que engloba todos los aspectos del significado no incluidos en la referencia. Más recientemente, Moulines afirma:

Mi interés primordial está en el sentido de los términos teóricos primitivos en las ciencias empíricas establecidas; en ese contexto, dentro del sentido de los términos caben, según creo, diversas cosas y entre ellas algo así como los "usos" wittgensteineanos -ya sólo sea porque para determinar el referente de, pongamos por caso, el término "masa", hay que hacer uso de una balanza que ha de funcionar de acuerdo con ciertas reglas "negociadas" por la scientific community- $y$ aqui nos salta a la cara la pragmática.

En cuanto a la interpretación wittgensteiniana de "sentido" fregeano, aunque la idea resulta novedosa e interesante, no deja de ser una posición que se opone a gran parte de las interpretaciones estándares. En una línea de interpretación de Frege, se afirma que podemos concebir el sentido fregeano en términos de conocimiento compartido, que nos permite la identificación del referente. Así, cuando Frege sostiene que "el sentido es el valuarte más preciado de la humanidad", la interpretación bastante generalizada, asocia "sentido" con "saber que", que en Frege se relaciona con la idea de saber objetivo, lógico (Frege, 1892, 1918). Pero una lectura pragmática, implica una interpretación ligada a la idea de "saber como".

Tal vez, se podría optar por una conceptualización tripartita del significado, sumando a la categorización fregeana algún término nuevo, o usar otro término con mayor abarque, que incluya "sentido" fregeano, pero agregue el componente pragmático. Lo que me parece es que el "sentido" fregeano no puede interpretarse pragmáticamente sin que se convierta en algo no-fregeano.

6. Consideraciones finales. En los puntos anteriores hemos realizado un esbozo de algunos aspectos de la ontosemántica en la $\mathrm{CE}$, en particular la distinción T-teórico, 
T-no-teórico, y la determinación del significado de los términos científicos.

Hemos comentado antes que nos interesa evaluar los límites de las soluciones programáticas de la CE a estos problemas. En relación a la propuesta de Sneed (1971) hemos señalado las nociones pragmáticas involucradas en la distinción aludida. Entre éstas, hemos comentado el rol central de las nociones de "determinación" y "métodos de determinación" de los términos teóricos; y las nociones de "presuposiciones" y de "disponer de una teoria". Asimismo, hemos enfatizado el rol de la concepción de "intended aplications", que implica la idea de que ciertas estructuras se aplican a cierto dominio empírico, de acuerdo a una concepción pragmático-intencional de aplicación. Finalmente, respecto de este aspecto, la relación entre la estructura formal y el conjunto de aplicaciones intencionales se presenta con un carácter instrumental y convencional: nuestras construcciones modelo-teóricos pueden servirnos para la descripción y explicación de cierto sistema fenoménico, a los que se aplica, según objetivos de la comunidad científica en circunstancias histórico-pragmáticas determinadas.

Stegmüller $(1973,1976)$ mostraba un claro interés filosófico por profundizar y desarrollar las implicancias del enfoque pragmático presente en Sneed (1971), tanto en relación a los aspectos epistémicos, como a los actitudinales en la ciencia y la actividad científica. Sin embargo, a pesar de su interés manifiesto en la elucidación metateórica de aquellas nociones pragmáticas, éstas permanecieron sin un tratamiento posterior más exhaustivo.

A partir de Balzer \& Moulines (1980) la ampliación del marco metateórico para el tratamiento de la distinción entre términos T-teóricos y T-no-teóricos ha permitido mayor precisión en el tratamiento. Asimismo, la posición seguida luego en Moulines (1985) representó una profundización y clarificación de la propuesta original de Sneed y Stegmüller.

Como se ha comentado, la postura moulinesiana posterior respecto de la determinación del significado de los términos científicos presenta un acercamiento de la pragmática wittgensteiniana. Vimos que en la interpretación propuesta por el autor se asume que, a pesar de que la concepción fregeana de referencia está en la base de la concepción semántica de las teorias científicas, es necesario reconocer aspectos no-referenciales del significado. Aspectos no referenciales no pueden identificarse sin más a la noción de "sentido" fregeano, y esto lo deja muy en claro Moulines. Se trata más bien de ampliar la semántica fregeana a campos pragmáticos, para lo cual la noción wittgensteniana de "uso" parece resultar una vía prometedora. Sin embargo, creo que la interpretación wittgensteiniana de "sentido" fregeano aportada por Moulines, por los motivos antes expuestos, resulta difícil de sostener, aunque tal vez se requeriría de tratamientos futuros, que permitan seguir elucidando esta propuesta.

En términos generales, la vía señalada en la CE respecto de la necesidad de atender a los componentes pragmáticos ligados al criterio de teoricidad, especialmente en los relativos al rol de la idea de aplicaciones intencionales (y en particular, aplicaciones paradigmáticas), es sin duda, el aporte fundamental en la elucidación metateórica del significado de los términos científicos. Sin embargo, la propuesta presenta limitaciones: (a) el desarrollo de los componentes pragmáticos ha sido escasamente desarrollada; (b) parte de la propuesta resulta tradicional, y se halla aún en parte circunscripta a una imagen de la ciencia como actividad asertórico-representacional.

En relación a (a) estimo que la solución pragmática a que hemos aludido arriba fue programáticamente prometedora. Tanto la concepción originaria de Sneed, como la de Stegmüller sobre los términos científicos contenían un conjunto de nociones pragmáticas, entendiendo por tales, nociones relativas a las prácticas científicas, que posibilitaron un diálogo fecundo entre la CE y las ideas de Kuhn $(1962,1976)$ de ciencia normal, comunidad científica, etc., como también con la concepción de Hempel (1973); sin embargo, esas nociones no tuvieron un tratamiento sistemático, ni recibieron una mayor profundización. El camino posterior de la CE -aun a pesar de los interesantes aportes metateóricos de Moulines- siguió una vía de clarificación metateórica que conllevó un aumento de la formalización, que si bien ayudó a ganar en precisión, fue lentamente dejando fuera de la agenda metateórica la elucidación de las nociones pragmáticas programáticamente introducidas en los comienzos.

Clarificando (b) podría agregar que la propuesta resulta tradicional, en el sentido en que la disponibilidad de 
una teoría se piensa en relación a la posibilidad de hacer aserciones teóricas acerca de $\mathrm{X}$, donde $\mathrm{X}$ debe amoldarse (modelarse) según $\mathrm{T}$ (aunque al mismo tiempo debe poder ser de algún modo modelable en un lenguaje diferente a T). De modo que, la noción de "disponer de una teoría T" está directamente asociada a la idea de realizar aserciones empíricas de $\mathrm{T}$.

Finalmente, cabe agregar que la propuesta moulinesiana de extender la noción de "sentido" fregeano Wittgeinstein dixit a una pragmática, es sin duda mucho menos abar- cadora que la propuesta original de Sneed y Stegmüller. Esta salida se circunscribe al campo de la pragmática del significado; pero deja de lado muchos otros aspectos que serian parte de una agenda de problemas del enfoque pragmático sobre la determinación de los términos en la ciencia, rasgos que han sido ubicados en una dimensión praxista del análisis de la ciencia. Sin embargo, los planteos de Moulines, a pesar de las aristas restrictivas expuestas, constituyen los aportes contemporáneos más significativos al tratamiento del problema en el marco de la tradición estructuralista de la ciencia.

\section{NOTAS}

1 Dummett ha comparado la revolución que Frege provocó en la tradición filosófica con la que Descartes habia provocado tres siglos antes: "Quizá el aspecto más importante de la revolución de Descartes en la filosofía fue dar este lugar central a la teoria del conocimiento, lugar que nunca había ocupado con anterioridad. Desde el tiempo de Descartes hasta hace muy poco, la pregunta básica para la filosofía era qué podemos conocer y cómo podemos justificar nuestras pretensiones de conocimiento; y el problema filosófico fundamental era hasta dónde puede refutarse el escepticismo y qué tanto debe aceptarse. Frege fue el primer filósofo posterior a Descartes que rechazó totalmente esta perspectiva [...] Para Frege, el principio de la filosofía era la lógica" Dummet (1990, 158-159).

2 La noción de "disponer de una teoría" había sido sugerida ya por Sneed (1971) en relación a las nociones kuhnianas de comunidad científica, $y$ de ciencia normal. Este enfoque se desarrolló más ampliamente en Stegmüller (1973, 1976).

Recibido: 13 de junio de 2009

Aceptado: 13 de diciembre de 2009
3 En la misma línea se proseguirian los trabajos de Moulines (1992, 1998).
4 Como dice el mismo Moulines, se trata en verdad de la negación del CT', o de una versión más sofisticada del mismo: un término es T-no-teórico si y sólo si no es T-teórico en el sentido de CT' (Moulines, 1985, 477).

5 Cf. Wittgenstein, Ludwig: Investigaciones filosóficas, Parte I, $\$ 41$ (citado en Moulines, 2002, 1).

6 Los textos de Moulines transcriptos en itálicas son textos inéditos, que forman parte de comunicaciones escritas mantenidas con el autor (abril/ junio 2002).

\section{BIBLIOGRAFÍA}

Balzer, Wolfgang (1986): "Theoretical Terms: A New Perspective", The Journal of Philosophy, 83: 71-90.

Balzer, Wolfgang y C. Ulises Moulines (1980): "On Theoreticity", Synthese, 44(3): 467-494.

Balzer, Wolfgang, C. Ulises Moulines y Joseph D. Sneed (1987): An Architectonic for Science, Dordrecht: Reidel.

Balzer, Wolfgang y C. Ulises Moulines (eds.) (1996): Structuralist Theory of Science, Berlin-New York: Walter de Gruyter.

Coffa, Alberto (1991): "La filosofía de la ciencia después de Kuhn", Cuadernos de Filosofía, 35: 7-23. 
Dummet, Michael (1990): "La filosofía de Frege", en Dummet, Michael, La verdad y otros enigmas, México: Fondo de Cultura Económica.

Frege, Gottlob (1892): "Sobre sentido y referencia", en Estudios sobre semántica, Barcelona: Ariel, 1984.

Frege, Gottlob (1918): "El pensamiento: una investigación lógica", en Investigaciones Lógicas, Madrid: Tecnos, 1984.

Hempel, Carl Gustav (1973): "El significado de los términos teóricos: una crítica de la concepción empirista estándar", en Olivé, León y Ana Rosa Pérez Ransanz (comp.), Filosofía de la ciencia: teoría y observación, México: Siglo XXI, 1989.

Gähde, Ulrich (1983): T-Theoretizität und Holismus, Frankfurt/Main-Bern: Peter Lang.

Gähde, Ulrich (1990): "On Innertheoretical Conditions for Theoretical Terms", Erkenntnis, 32: 215-33.

Kuhn, Thomas S. (1962): La estructura de las revoluciones científicas, México: Fondo de Cultura Económica, 1991.
Kuhn, Thomas S. (1976): "Theory-Change as Structure-Change: Remarks on the Sneed Formalism", Erkenntnis, 10: 179-199.

Moulines, C. Ulises (1982): Exploraciones metacientificas, Madrid: Alianza.

Moulines, C. Ulises (1984): "Ontological Reduction in the Natural Sciences", en Balzer, Wolfgang, Pearce, David A. y Heinz-Jürgen Schmidt (eds.), Reduction in Science, Dordrecht: Reidel.

Moulines, C. Ulises (1985): "Los términos teóricos y los principios puente: una crítica de la (auto)crítica de Hempel", en Olivé, León y Ana Rosa Pérez Ransanz (comp.), Filosofía de la ciencia: teoría y observación, México: Siglo XXI, 1989.

Moulines, C. Ulises (1991): Pluralidad y recursión. Estudios Epistemológicos, Madrid: Alianza.

Moulines, C. Ulises (1994): “¿ué clases de cosas hay?", en Gómez Pin, Víctor (ed.), Categorías e inteligibilidad global, Barcelona: Universidad Autónoma de Barcelona.
Moulines, C. Ulises (1998): "Esbozo de ontoepistemosemántica", Theoria, 13(1): 146-159.

Moulines, C. Ulises (2002): "Ontosemántica en perspectiva estructuralista", III Encuentro Iberoamericano sobre Metateoría Estructural, Granada, España (inédito).

Sneed, Joseph (1971): The Logical Structure of Mathematical Physics, Dordrecht: Reidel.

Stegmüller, Wolfgang (1973): Probleme und Resultate der Wissenschaftstheorie und Analytischen Philosophie, Bd. II: Theorie and Erfahrung, Hlbd. II: Theorienstrukturen und Theoriendynamik, Wien-New York: Springer.

Stegmüller, Wolfgang (1976): "Cambio teórico accidental (no sustancial) y desplazamiento de teorias", en AA.W. Estructura y desarrollo de las teorías científicas, México: UNAM, 1986.

Toumela, Raimo (1973): Theoretical Concepts, Wien-New York: Springer. 